\title{
Psychological Factors Influence the Symptoms of Gastroesophageal Reflux Disease
}

TO THE EDITOR: I read with interest the study by Lee et al ${ }^{1}$ regarding the relationship between existence of typical symptoms and psychological factors in patients with erosive esophagitis. The study was conducted by enrolling 29 symptomatic and 26 asymptomatic patients with erosive reflux disease (ERD). They used the Symptom Checklist-90-Revision (Korean version, SCL-90-R) ${ }^{2}$ to identify the presence of psychological symptoms. In the SCL-90-R, each of the items is rated on a 5-point scale of distress (0-4) ranging from "not at all" to "extremely." The 9 primary symptom dimensions are labeled as: somatization (SOM), obsessive-compulsive behavior (O-C), interpersonal sensitivity (I-S), depression, anxiety, hostility, phobic anxiety (PHOB), paranoid ideation and psychoticism (PSY). There are 3 global indices as well: the global severity index is a mean score of all 90 items; the positive symptom distress index; the positive symptom total, which represents the number of symptoms rated as $\geq 1$. The authors demonstrated that symptomatic patients with ERD were more likely to have higher scores on psychological symptoms (SOM, O-C and $\mathrm{PHOB}$ ) than asymptomatic patients with ERD. The authors should be congratulated for completing study, which supports the assumption that psychological factors influence the development of symptoms in patients with gastroesophageal reflux disease (GERD). Nevertheless, I would like to consider 2 concerns for the accurate understanding of the meaning of these results.

First, the significant difference in the score of SOM, O-C and $\mathrm{PHOB}$ between them might be rather statistically different. In Lee's study, ${ }^{1}$ the mean values for SOM, O-C and PHOB were 52.37, 46.22 and 49.59, in symptomatic ERD group respectively. I wonder whether the scores of SOM, O-C and $\mathrm{PHOB}$ in symptomatic ERD are higher than those in general population. Nunez-Rodriguez and Miranda Sivelo ${ }^{3}$ conducted the study using the SCL-90-R to determine association between psychological factors and GERD. In the study, the scores of SOM, O-C, I-S, depression, anxiety, hostility, $\mathrm{PHOB}$, paranoid ideation and PSY were 120/55, 79/60, 64/45, 81/72, 57/52, 42/45, 37/25, 55/47 and 40/21 in GERD group and Spanish healthy population, respectively. GERD subjects had higher scores compared to healthy controls on SOM, O-C, I-S, PHOB, and PSY. Thus Lee's study might be able to provide the SCL-90-R data of healthy controls to give us a better overview of association between psychological factors and GERD symptom.

Second, it is unclear whether the psychological dimensions occurred before the reflux symptoms and played an important role in the development of GERD symptoms. The psychological diseases may be a consequence of reflux symptoms. If the authors reanalyze the SCL-90-R data before and after proton pump inhibitor treatment, it would be helpful in understanding whether the GERD symptoms are influenced by psychological factors or not.

Tae Hee Lee Institute for Digestive Research, Soonchunhyang University College of Medicine, Seoul, Korea

1. Lee SP, Lee KN, Lee OY, et al. The relationship between existence of typical symptoms and psychological factors in patients with erosive esophagitis. J Neurogastroenterol Motil 2012;18:284-290.

2. Kim KI, Kim JH, Won HT. Korean manual of Symptom Checklist90-Revision. Seoul: Choong Ang Aptitude Publishing Co. 1984:139.

3. Núñez-Rodríguez MH, Miranda Sivelo A. Psychological factors in gastroesophageal reflux disease measured by scl-90-R questionnaire. Dig Dis Sci 2008;53:3071-3075.

Conflicts of interest: None. 